\title{
New methodology to assess activity status of occlusal caries in primary teeth using laser fluorescence device
}

\author{
Mariana Minatel Braga \\ Departmento de Ortodontia e Odontopediatria \\ Faculdade de Odontologia da São Paulo \\ São Paulo, Brazil 05508-000
}

\author{
Monique Saveriano de Benedetto \\ Universidade de São Paulo \\ Faculdade de Odontologia \\ São Leopoldo Mandic, Brazil 13100-000
}

\author{
Jose Carlos Pettorossi Imparato \\ Fausto Medeiros Mendes \\ Universidade de São Paulo \\ Departmento de Ortodontia e Odontopediatria \\ Faculdade de Odontologia da São Paulo \\ São Paulo, Brazil 05508-000
}

\begin{abstract}
An in vivo study was conducted to verify the ability of laser fluorescence (LF) to assess the activity status of occlusal caries in primary teeth, using different air-drying times. Occlusal sites (707) were examined using LF (DIAGNOdent) after air-drying for $3 \mathrm{~s}$ and $15 \mathrm{~s}$, and the difference between readings (DIF15 s-3 s) was calculated. For concurrent validation of LF, visual criteria-Nyvad (NY) and Lesion Activity Assessment associated with the International Caries Detection and Assessment System (LAA-ICDAS)-were the reference standards for lesion activity. Histological exam using a $\mathrm{pH}$-indicator dye $(0.1 \%$ methyl red) was performed in 46 exfoliated/extracted teeth for criterion validation. LF readings and DIF15 s-3 s were compared using Kruskall-Wallis and Mann-Whitney tests. Receiver operating characteristic analyses were performed and validity parameters calculated, considering the caries activity assessment. Using NY, active lesions (3 s: $30.0 \pm 29.3 ; 15$ s: $34.2 \pm 30.6$ ) presented higher LF readings than inactive lesions (3 s: $17.0 \pm 16.3 ; 15 \mathrm{~s}$ : 19.2 $\pm 17.3 ; p$ $<0.05)$, different from LAA-ICDAS. Active cavitated caries resulted in higher LF readings (3 s: $50.3 \pm 3.5 ; 15 \mathrm{~s}: 54.7 \pm 30.2$ ) than inactive cavitated caries (3 s: $19.9 \pm 16.3 ; 15$ s: $22.8 \pm 16.8$ ). Therefore, LF can distinguish cavitated active and inactive lesions classified by NY, but not by LAA-ICDAS; however, this difference might be related to the visual system rather than to LF. The air-drying time could be an alternative to improve the caries activity assessment; however, longer air-drying time is suggested to be tested subsequently. () 2010 Society of Photo-Optical Instrumentation Engineers. [DOI: 10.1117/1.3463007]
\end{abstract}

Keywords: dental caries; activity assessment; laser fluorescence; drying; criterion validity; concurrent validity.

Paper 10073RR received Feb. 12, 2010; revised manuscript received May 7, 2010; accepted for publication May 17, 2010; published online Jul. 29, 2010.

\section{Introduction}

Caries activity is an important factor to consider in clinical decision-making due to its relationship with lesion prognosis or possibility of progression/regression. ${ }^{1}$ Thus, there is a trend toward significantly increasing interest in investigating this topic in future. Nowadays, the only method available to assess caries lesion activity is visual inspection. Despite being a practical and easy method for clinicians to use, ${ }^{2}$ visual inspection is subjective and strongly dependent on the examiner's decision. The association of other objective and quantitative diagnostic methods is therefore desirable in order to improve their reliability and allow more precise monitoring of lesions.

Laser fluorescence (LF) is a diagnostic method that has shown promising results in caries detection, especially because of its reproducibility. ${ }^{3}$ The LF device consists of a diode laser emitting a light $(\lambda=655 \mathrm{~nm})$ that is absorbed by dental

Address all correspondence to Mariana Minatel Braga, Faculdade de Odontologia da Universidade de São Paulo, Av. Lineu Prestes, 2227, São Paulo, SO Brazil 05508-000. Tel: 55-1-3091-7835; Fax: 55-11-3091-7854; E-mail: mmbraga@usp.br. tissues and is partially reemitted as a near-infrared fluorescence. The system collects this fluorescence and provides quantitative measures on a scale from 0 to 99 . The higher the number, the deeper the caries lesion. ${ }^{4}$ The fluorescence read by the device is probably from bacterial metabolic byproducts, especially porphyrines. ${ }^{4-6}$ As active lesions are more infected than inactive lesions, ${ }^{1}$ LF could be a method for use as an adjunct in caries activity assessment.

Some previous in vivo studies have shown that it is possible to distinguish active and inactive lesions in smooth ${ }^{7}$ and occlusal surfaces. ${ }^{8,9}$ Nevertheless, some inactive caries, especially on occlusal surfaces, present brownish or dark staining, ${ }^{8,10,11}$ which could interfere in caries diagnosis, since LF readings are generally high for this type of lesion. ${ }^{12,13}$ Consequently, LF ability to assess caries activity appears to be contradictory.

Previous studies have shown that long air-drying time could influence LF readings. ${ }^{14}$ We hypothesized that LF readings, after longer air-drying time, would increase more sig-

1083-3668/2010/15(4)/047005/7/\$25.00 @ 2010 SPIE 
nificantly in active caries lesions than in inactive lesions. This occurrence would be related to the higher concentration of bacterial metabolites in active lesions, which would become more concentrated after water loss. In addition, active lesions are expected to be more porous and contain more water inside. Consequently, after air-drying, the active lesions would present more differences than inactive ones. These phenomena would allow active and inactive caries to be distinguished more objectively. Similar methodology has already been applied to another fluorescence method (quantitative lightinduced fluorescence-QLF). ${ }^{15,16}$ Nevertheless, this diagnostic method is based on a caries detection principle more related to mineral loss, which differs from LF. Consequently, testing this methodology for LF is extremely relevant.

Based on these assertions, the aim of this in vivo study was to verify the ability of LF to assess the activity status of occlusal caries with the similar depths in primary teeth, when using different air-drying times.

\section{Material and Methods}

The protocol of this study was approved by the Ethics Committee of the School of Dentistry, University of São Paulo.

\subsection{Subject Selection}

One hundred and sixty-five children, aged 3 to 12 years, who sought dental treatment, with at least one primary molar to be examined were invited to participate in the study. One hundred and seventeen children $(7.3 \pm 2.5$ years old $)$ completed the clinical phase of the study (positive response rate of $84.2 \%$ ). We selected children in the city of Araras, Brazil (67 children), or at the School of Dentistry, University of São Paulo, Brazil (50 children). Both cities have had $0.7 \mathrm{mg} / \mathrm{L} \mathrm{F}^{-}$ in their water supply since 1996. Written, informed consent was obtained from the children's parents or guardians.

For each child, all fully erupted primary molar teeth were examined. Teeth with restorations, hypoplastic defects, sealants, or frank cavitation were excluded from the study sample. A total of 707 primary molar teeth were included in the study. For each tooth, the site on the occlusal surface more prone to be carious or that had the most advanced lesion was selected as the investigation site, and this was marked on a diagram on a specially designed form.

\subsection{LF Examination}

A single, trained examiner (MMB), expert in using the LF device, performed measurements of the selected teeth. All examinations were carried out in a dental unit using an operating light for illumination and a 3-in-1 syringe.

A LF device, DIAGNOdent (KaVo, Biberach, Germany), was used in examinations. For each child, the device was calibrated against a ceramic standard. After that, it was calibrated on the middle of the buccal surface of every tooth before the examination of each occlusal surface. Last, tip A was placed on a previously selected site and rotated around a vertical axis. Two measurements were performed consecutively for each site, and the mean value was calculated.

For each surface, two assessments were made, following the preceding methodology. The first was made after airdrying for $3 \mathrm{~s}$ (Ref. 14) followed by the child waiting in the dental unit for $5 \mathrm{~min}$ in order to rehydrate teeth, and then the assessment was repeated, but now after air-drying for $15 \mathrm{~s}$.

\subsection{Concurrent Validation (Visual Examination)}

The concurrent validation of LF readings with regard to caries activity assessment was performed by visual examination based on two different visual scoring criteria: Nyvad criteria $(\mathrm{NY})^{11}$ and International Caries Detection and Assessment System (ICDAS) as an adjunct to the Lesion Activity Assessment associated with the International Caries Detection and Assessment System (LAA-ICDAS). ${ }^{17}$ These systems are based on visual inspection after prolonged air-drying. The examiners were supposed to evaluate surfaces concerning the presence of opacities and discolorations, discontinuities of surface integrity, occurrences of cavities, and exposure of dentine. After using the indices, the examiners are able to classify the caries lesions with regard to severity and activity status but using a slight different rationale (Fig. 1).

Two independent and expert examiners (MMB and FMM) were previously trained and calibrated to perform both visual criteria, as described in a previous study, ${ }^{18}$ and afterward scored all the selected teeth $(n=707)$ according to the visual criteria. Before examinations, the teeth were carefully cleaned with a rotating bristle brush and pumice/water slurry. Visual inspection was performed according to a standardized methodology, with subjects seated in a dental unit, under operating light illumination, and the examiner using a 3-in-1 syringe, flat buccal mirror, and World Health Organization (WHO) periodontal probe. Air-drying was performed for $5 \mathrm{~s}$. After this, the clinical evaluation followed in accordance with each criterion. Both indices were used and compared with LF measurements independently.

First, one system was used for each subject. In another appointment, the examiners blindly reexamined the investigation sites using the system that had not been used the first time. The order in which each system was used for each child was randomly selected. In case of disagreement between the examiners after using each criterion independently, a consensus was reached after reexamination of the investigation sites and discussion. The final score used in the subsequent analyses was the consensus score for both NY and LAA-ICDAS criteria.

Visual examinations were performed two weeks after the LF measurements had been taken in the child now being examined, and the examiner was blind to the previous LF readings.

\subsection{Criterion Validation (Histology)}

A compound subsample consisting of 46 exfoliated or extracted primary molars, obtained from the full sample, was collected within 2 weeks after the clinical recordings, and the teeth were stored frozen at $-20^{\circ} \mathrm{C}$ up to 1 month. ${ }^{19,20}$ These teeth were hemisectioned using a diamond saw (Microdont, São Paulo, Brazil) in the location of the selected site. Both sides of each section were examined by two examiners (MMB and FMM) blind to each other and to the clinical visual scores and LF values, using a stereomicroscope at 8 to $20 \times$ magnification and reflected light (SZPT Olympus, Tokyo, Japan).

With regard to depth validation, a 5-point scale was used to judge the lesion depth: D0—no caries; D1—caries lesion lim- 

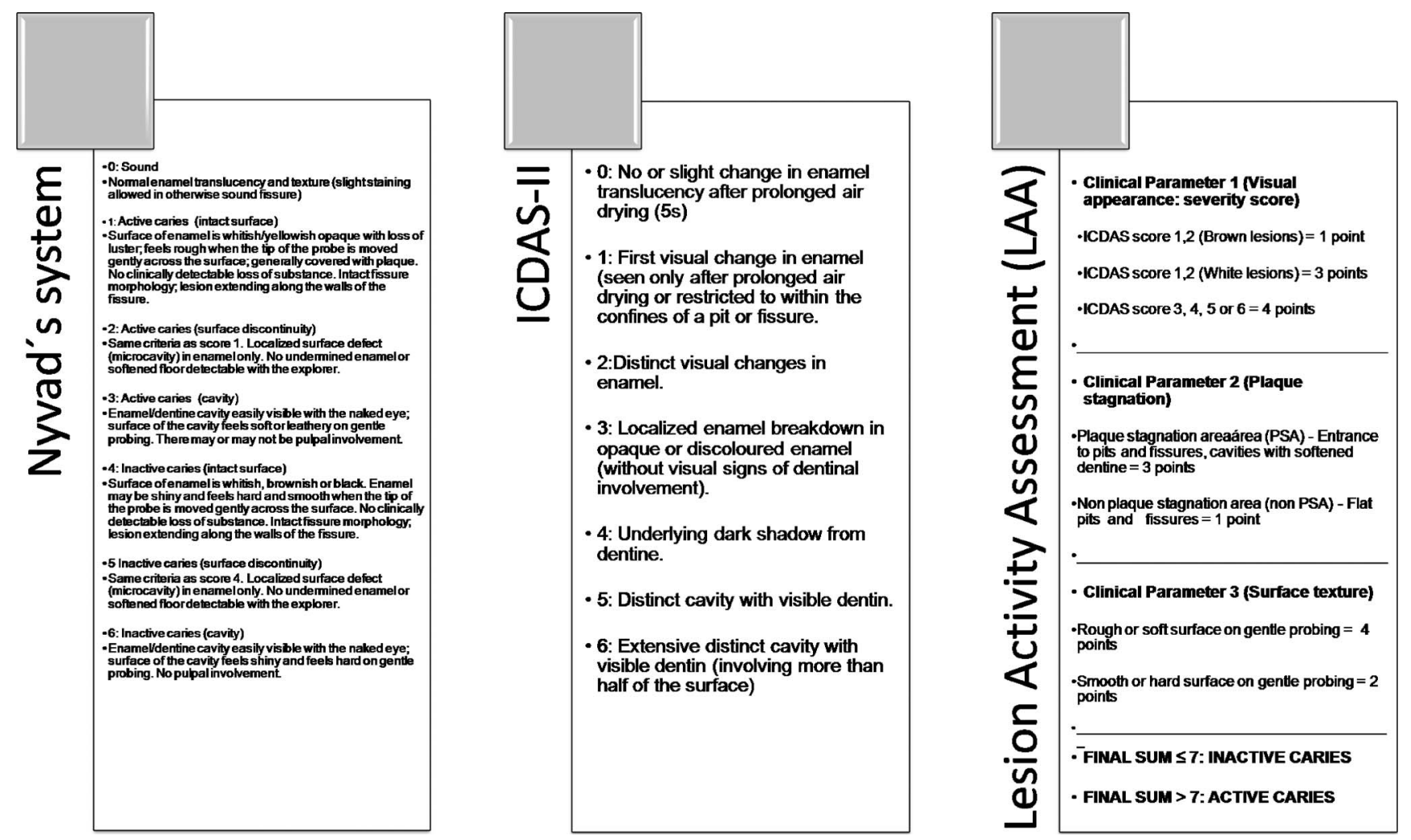

Fig. 1 Criteria used in the visual examination (concurrent validity) —NY and ICDAS+LAA

ited to the outer half of the enamel; D2 - caries extending into inner half of the enamel but not to the amelo-dentinal junction; D3 - caries limited to the outer half of the dentin; D4caries involving the inner half of the dentin. The deepest score in relation to each investigation site was used in the subsequent analyses. In cases in which disagreement between examiners occurred, new examinations were performed, and a consensus decision was reached. This process took less than $5 \mathrm{~min}$.

Immediately after assessing the depth of the lesion, a $0.1 \%$ methyl red solution (Aldrich, Milwaukee) was dripped onto the two sectioned faces of the same teeth mentioned earlier, in order to validate caries activity. After one minute, the excess of the solution was removed, and the sections were evaluated in the same manner as used for the lesion depth assessment, but at 8 to $50 \times$ magnification. Section faces in which the lesion appeared red or reddish were classified as active. Yellow-stained sections were classified as inactive. ${ }^{10,21}$

\subsection{Statistical analysis}

As LF reading distribution was not normal (Anderson-Darling test), nonparametric tests were used. For the analysis, three different outcomes were considered: mean of LF readings after air-drying for $3 \mathrm{~s}$; mean of LF readings after air-drying for $15 \mathrm{~s}$, and mean of the difference between values obtained after air-drying for $15 \mathrm{~s}$ and $3 \mathrm{~s}$ (DIF15 s-3 s).

For full sample analysis, the examined sites $(n=707)$ were divided into three different groups: sound sites, inactive lesions, and active lesions according to both visual criteria (NY and LAA-ICDAS). The LF readings were compared among these groups using the Kruskal-Wallis test. The active and inactive noncavitated lesions as well as active and inactive cavitated lesions were compared by the Mann-Whitney test. For this division, both visual criteria were also considered independently.

Using the compound subsample of 46 extracted or exfoliated teeth, receiver operator characteristic (ROC) analysis was performed to verify LF performance in caries activity assessment. The reference method was the histology associated with the $1 \%$ methyl red dye. Two outcomes were considered: sound sites + inactive lesions versus active lesions and inactive lesions versus active lesions (excluding sound sites). All calculations were made using both air-drying times and DIF15 s-3 s. From all ROC analysis, the cut-off points for each threshold and area under ROC curves were obtained. Based on these cut-off points, sensitivity, specificity, accuracy, and positive and negative predictive values were calculated for each specific condition mentioned earlier. These validity parameters were compared among different thresholds for depth detection using McNemar (sensitivity, specificity, and accuracy) and chi-square test (predictive values). In order to compare LF sensitivity, specificity, and accuracy in assessing caries activity after different air-drying times and the DIF15 s-3 s, the McNemar test was used. For comparison of LF positive and negative predictive values for the same purpose, the chi-square test was used.

For all statistic analyses, the level of significance was $p$ $<0.05$. 
Braga et al.: New methodology to assess activity status of occlusal caries in primary teeth...

Table $1 \mathrm{LF}$ readings (mean \pm SD) after air-drying for $3 \mathrm{~s}$ and $15 \mathrm{~s}$ and difference between 15 -s and 3-s readings (DIF $15 s-3$ s) in teeth examined visually by NY.

\begin{tabular}{|c|c|c|c|}
\hline & LF $3 \mathrm{~s}$ & LF $15 \mathrm{~s}$ & DIF $15 s-3 s$ \\
\hline \multicolumn{4}{|c|}{ Noncavitated lesions (scores 1 and 4) } \\
\hline Inactive (85) & $16.5 \pm 16.4$ & $18.4 \pm 17.4$ & $2.0 \pm 5.5$ \\
\hline Active (114) & $16.4 \pm 17.8$ & $20.5 \pm 21.7$ & $4.1 \pm 8.3^{a}$ \\
\hline \multicolumn{4}{|c|}{ Cavitated lesions (scores 2, 3, 5, and 6) } \\
\hline Inactive (25) & $19.9 \pm 16.3$ & $22.8 \pm 16.8$ & $2.9 \pm 5.3$ \\
\hline Active (77) & $50.3 \pm 3.5^{a}$ & $54.7 \pm 30.2^{a}$ & $4.5 \pm 8.5$ \\
\hline \multicolumn{4}{|c|}{ All samples (scores 0 to 6 ) } \\
\hline Sound (406) & $3.9 \pm 6.6 a$ & $4.6 \pm 7.5 a$ & $0.6 \pm 2.1 \mathrm{a}$ \\
\hline Inactive lesions ( 110$)$ & $17.0 \pm 16.3 b$ & $19.2 \pm 17.3 b$ & $2.2 \pm 5.4 b$ \\
\hline Active lesions (191) & $30.0 \pm 29.3 c$ & $34.2 \pm 30.6 c$ & $4.2 \pm 8.4 \mathrm{c}$ \\
\hline \multicolumn{4}{|c|}{$\begin{array}{l}{ }^{a} \text { Statistically significant difference by the Mann-Whitney test }(p<0.05) \text { in com- } \\
\text { parison with the row above. }\end{array}$} \\
\hline
\end{tabular}

\section{Results}

\subsection{Entire Sample}

Using NY as a reference for activity assessment, among noncavitated lesions, LF readings from active caries were similar to those from inactive caries. However, the difference between these measurements was slightly higher for active lesions. Oppositely, in the cavitated sample, the mean DIF15 s-3 s was similar, irrespective of caries activity status. On the other hand, active cavitated lesions resulted in higher readings when compared with inactive lesions. With regard to the entire sample, $\mathrm{LF}$ readings distinguished active lesions from inactive lesions and from sound sites. Similarly, the DIF15 s-3 s distinguished active lesions from inactive lesions and sound sites, as well as inactive lesions from sound sites (Table 1).

With reference to LAA-ICDAS, no differences were noted between LF measurements of active and inactive lesions, irrespective of surface integrity or air-drying time. The same trend was observed for DIF15 s-3 s. Including sound teeth, the LF readings were able to differentiate sound sites from carious sites in general, but not specifically active from inactive lesions (Table 2).

\subsection{Compound Subsample}

With regard to activity assessment, the LF sensitivities $(0.54$ $0.92)$, specificities (0.64-0.94), positive predictive values (0.50-0.88), and negative predictive values (0.60-0.95) varied from moderate to high depending on the air-drying times. The same was observed for the LF accuracy, that was considered as the number of correct diagnosis (0.69 to 0.87-Table 3$)$.
Table $2 \mathrm{LF}$ readings (mean \pm SD) after air-drying for $3 \mathrm{~s}$ and $15 \mathrm{~s}$ and difference between 15 -s and 3 -s readings (DIF15 s-3 s) in teeth examined visually by LAA-ICDAS.

\begin{tabular}{|c|c|c|c|}
\hline & LF $3 \mathrm{~s}$ & LF $15 \mathrm{~s}$ & DIF $15 s-3 s$ \\
\hline \multicolumn{4}{|c|}{ Noncavitated lesions (scores 1 and 2) } \\
\hline Inactive (51) & $17.3 \pm 17.3$ & $19.8 \pm 18.9$ & $2.4 \pm 6.2$ \\
\hline Active (162) & $15.4 \pm 16.7$ & $18.7 \pm 19.8$ & $3.3 \pm 7.4$ \\
\hline
\end{tabular}

Cavitated lesions (scores 3 to 6 )

$\begin{array}{llll}\text { Inactive (3) } & 41.3 \pm 34.0 & 48.7 \pm 31.7 & 7.3 \pm 8.4 \\ \text { Active }(100) & 43.4 \pm 30.9 & 47.3 \pm 30.7 & 4.0 \pm 7.9\end{array}$

All samples (scores 0 to 6 )

\begin{tabular}{cccc} 
Sound (391) & $3.9 \pm 6.5 \mathrm{a}$ & $4.5 \pm 7.3 \mathrm{a}$ & $0.5 \pm 2.1 \mathrm{a}$ \\
& & & \\
Inactive lesions (54) & $18.7 \pm 18.9 \mathrm{~b}$ & $21.4 \pm 20.4 \mathrm{~b}$ & $2.7 \pm 6.3 \mathrm{a}, \mathrm{b}$ \\
Active lesions (262) & $26.1 \pm 26.8 \mathrm{~b}$ & $29.6 \pm 28.2 \mathrm{~b}$ & $3.8 \pm 7.6 \mathrm{~b}$ \\
\hline
\end{tabular}

* Statistically significant difference by the Mann-Whitney test $(p<0.05)$ in comparison with the row above.

Note: Different letters show statistically significant differences between values in the same column, according to Kruskal-Wallis test. Numbers in parentheses refer to the samples $(n)$ in each category.

The LF validity parameters did not present statistically significant differences between different air-drying times and the DIF15 s-3 s when inactive lesions versus active lesions were considered. On the other hand, collapsing sound sites and inactive lesions, the specificity increased with higher air-drying time (15 s). The same was observed using the DIF15 s-3 s. In addition, sensitivity was reduced for DIF $15 \mathrm{~s}-3 \mathrm{~s}$ in comparison with air-drying for $3 \mathrm{~s}$ (Table 3 ).

\section{Discussion}

This study was conducted to test the validity of LF in caries activity assessment, and was based on two validation processes. First, concurrent validation was used, ${ }^{22}$ as other studies that focused on caries activity assessment by $\mathrm{LF}^{7,23}$ and also used visual inspection as a reference standard, since it is the only validated method capable of evaluating this. However, we used ranked scoring visual indices, previously validated clinically for activity assessment in primary teeth. ${ }^{24} \mathrm{Al}$ though it is not a perfect standard method, this methodology permits us to detect early caries lesions, assessing initial demineralization stages accurately. ${ }^{25}$ The option for using two visual systems is related to different activity assessment processes inherent in the two scoring criteria. Histology associated with methyl red dye, a $\mathrm{pH}$ indicator, ${ }^{10,21}$ was used in a subsample in order to check LF criterion validity, which has not yet been assessed for primary teeth.

Our results showed that LF was able to distinguish active and inactive occlusal lesions classified by NY in primary teeth. Previous studies have shown this to be possible in the smooth surfaces of permanent teeth. ${ }^{7,23}$ These previous studies, however, were not properly based on visual criteria for caries activity, despite using visual examination. 
Table 3 Cutoff points, sensitivity, specificity, accuracy (percentage of correct exams), area under ROC curves $(A z)$, and predictive values of LF considering (A) inactive lesions versus active lesions; (B) sound sites + inactive lesions versus active lesions, classified by histology associated with $1 \%$ methyl red dye $-n=46$.

\begin{tabular}{|c|c|c|c|c|c|c|}
\hline & \multicolumn{2}{|c|}{ LF $3 \mathrm{~s}$} & \multicolumn{2}{|c|}{ LF $15 \mathrm{~s}$} & \multicolumn{2}{|c|}{ DIF $15 s-3 s$} \\
\hline & $A$ & B & $A$ & B & $A$ & B \\
\hline Prevalence & $56.5 \%$ & $28 \%$ & $56.5 \%$ & $28 \%$ & $56.5 \%$ & $28 \%$ \\
\hline Cutoff point & 27 & 9 & 60 & 38 & 0 & 5 \\
\hline Sensitivity & $0.62 a$ & $0.92 a$ & $0.54 a$ & $0.62 a, b$ & $0.77 a$ & $0.39 \mathrm{~b}$ \\
\hline Specificity & $0.80 a$ & $0.64 a$ & $0.90 a$ & $0.94 \mathrm{~b}$ & $0.50 a$ & $0.85 a, b$ \\
\hline Accuracy & $0.74 a$ & $0.86 a$ & $0.69 a$ & $0.87 a$ & $0.65 a$ & $0.78 a$ \\
\hline $\mathrm{Az}$ & $0.64 a$ & $0.81 \mathrm{a}$ & $0.63 a$ & $0.80 a$ & $0.58 a$ & $0.56 \mathrm{~b}$ \\
\hline Positive predictive value & $0.80 a$ & $0.50 a$ & $0.88 a$ & $0.80 a$ & $0.67 a$ & $0.50 a$ \\
\hline Negative predictive value & $0.62 a$ & $0.95 a$ & $0.60 a$ & $0.86 a$ & $0.63 a$ & $0.78 a$ \\
\hline
\end{tabular}

Note: Different letters show statistically significant differences between values in the same row, considering the same categorization ( $a$ or $b$ ) $-p<0.05$.

When the LAA-ICDAS was adopted as a reference, no difference in LF readings was observed in active or inactive sites, different from NY. Different results observed using both indices can reflect a significant problem in one of the reference methods (visual). Although the process for evaluating sites has been standardized, examiners had to assess activity status in different ways: by choosing a single score that reflected a combination of features related to caries activity (NY) or by attributing different values to each characteristic with regard to activity status and making a mathematical calculation considering all of them (LAA-ICDAS). A previous study using these indices for activity assessment in primary teeth observed possible overestimation of caries activity by LAA-ICDAS, especially in cavitated lesions. ${ }^{24}$ Present findings can corroborate this statement, since 25 cavitated lesions were classified as inactive when NY was used, while only three lesions received the same classification using LAAICDAS.

Based on this, one could suppose that there may have been an interference resulting from using LAA-ICDAS as a reference standard in the LF validation. Probably, as suggested, ${ }^{24}$ if the LAA-ICDAS cut-off points were adjusted, different results could be found and this interference removed. Therefore, further studies applying the new cut-off points proposed should be developed.

Although different air-drying times were used ${ }^{14}$ as well as working with differences in LF readings after different airdrying times, as observed for other fluorescence devices, ${ }^{15,16}$ the possibility of distinguishing active and inactive lesions by LF in vivo was noted only when using NY as a reference (independent of surface integrity status). This difference corroborates the authors' hypothesis stated earlier with regard to overestimation of caries activity assessment when LAAICDAS was used. ${ }^{24}$
LF readings were only significantly different between cavitated active and inactive caries. In general, cavitated lesions presented a higher level of infection, ${ }^{26}$ which contributed to the presence of a higher amount of bacterial metabolites and, consequently, higher LF readings. If a cavitated lesion is active, more metabolites are constantly produced by oral microorganisms involved in lesion progression. Therefore, as observed, higher LF values had already been expected compared to inactive cavitated lesions, independent of the air-drying duration.

On the other hand, in spite of having a lower level of organic contents, noncavitated lesions could be distinguished by the difference between LF readings after $15 \mathrm{~s}$ and $3 \mathrm{~s}$ (DIF15 s-3 s). Although statistically significant, the magnitude of the difference was minimal. However, one can suppose that longer air-drying time could result in a more expressive variation. When lesions were dried for a longer time $(15 \mathrm{~s})$, the bacterial metabolites might tend to be concentrated, and a difference in fluorescence reading could be observed. As active lesions are more porous than inactive lesions, the air flow dessication can also result in changes of optical properties, especially light scattering, which would influence the fluorescence detected by the device. Scattering coefficient increases exponentially with increasing mineral loss. ${ }^{27}$ Active caries, which are less mineralized than inactive caries, are supposed to present high light scattering. In addition, when water evaporates from a caries lesion, light scattering occurs in air instead of water. Since the refractive index of air is lower than of water, the light scattering tends to be higher ${ }^{15}$ in an air-dried lesion. Due to the higher volume of pores in active caries lesions, a higher light scattering in active caries is comprehensible compared to inactive caries lesions. The increased backscattering increased the amount of 
remitted excitation light in the emission fibers used in the LF device, leading to an increase of the signal of LF detected.

The air-drying time ( $3 \mathrm{~s}$ or $15 \mathrm{~s}$ ) can influence LF performance in activity assessment, although different cut-off points for each situation are considered. Using air-drying for $15 \mathrm{~s}$, the method was better for identifying sound sites and inactive caries than active caries, since this modification in LF examination resulted in achieving moderate sensitivity and negative predictive values as well as increasing specificity. In fact, longer air-drying duration can improve the discrimination of active caries lesions. The LF device measures the organic alterations in caries, especially porphyrins. ${ }^{4,5}$ Thus, deeper or cavitated lesions, which are more infected ${ }^{26}$ would tend to result in higher LF readings and can interfere in the activity assessment using LF.

No previous study has evaluated LF performance in caries activity assessment. Compared with visual inspection methods, LF specificity was higher than that obtained with two visual indices. ${ }^{18,24}$ On the other hand, LF sensitivity can be higher than these indices or slightly lower, depending on the air-drying time. These findings corroborate the LF method as an adjunct to visual inspection for activity assessment, as observed for the caries detection and evaluation of caries lesions depth. $^{3}$

The increase in specificity for LF (15 s) observed when sound sites were included are related to the proportion of false positives in relation to true negative with regard to caries assessment status, since mostly sound sites were counted as true negatives. Nevertheless, the reduction in specificity was associated to a moderate sensitivity. This combination reinforces the hypothesis that active caries lesions, after being air-dried for $15 \mathrm{~s}$, are more prone to be distinguished from inactive caries or sound sites. Longer air-drying tends to increase LF readings more significantly for active caries lesions, as mentioned previously, and this fact can corroborate our earlier conclusions. With regard to clinical decision-making, this is an important feature, since each of these groups should be managed differently considering their prognosis. ${ }^{28}$

Both outcomes (with and without sound sites) were performed in order to considered two clinical situations. First, one intended to check whether LF could be used when the clinician is sure of the presence of the lesion but is in doubt about classifying the carious lesion as active or inactive. On the other hand, if the clinician is unsure about the presence as well as activity of the lesion, since the same treatment decision (no treatment at all) is used for inactive carious lesions and sound surfaces, analyses using a combination of sound surfaces and inactive caries lesions would be better. Based on our previous remarks, high LF specificity after 15-s air-drying in caries activity assessment can slightly increase the number of false negatives. Indeed, some active lesions could be classified as sound sites or inactive caries, as explained previously. For the patient, this false result could culminate in neglecting the use of a therapy for arresting caries. The association of a visual method to prevent this occurrence should be reinforced.

Analyzing the cut-off points for activity assessment, one could surmise that, considering only caries lesions, the DIF15 s-3 s could distinguish active caries from inactive caries. When there was not DIF15 s-3 s (cut-off=0), the lesion would be inactive. Besides, if DIF15 s-3 s was higher than zero, lesion would be active. As expected for low cut-off points, the sensitivity was high while specificity was low, which is not significant with regard to caries activity, since the clinician would plan actions to arrest the lesion. Indeed, it is preferable, in case of doubt, to diagnose as active and establish measures to control this lesion. ${ }^{11}$

On the other hand, according to our results, if one had a DIF15 s-3 s of more than five arbitrary units between two LF readings ( $3 \mathrm{~s}$ and $15 \mathrm{~s}$ ), we could classify the lesion as active. This cut-off point is, however, slightly different from that defined earlier and longer time of air-drying $(>15 \mathrm{~s})$ can be an alternative to find a more expressive variation, as mentioned previously. In addition, this different cut-off point could result from inclusion of sound surfaces. Although sound surfaces, do not require treatment for being arrested as do inactive caries, they generally present lower LF readings than inactive lesions. Therefore, it is reasonable that the DIF15 s-3 s was slightly higher when sound surfaces are included.

The longer air-drying as a manner to discriminate active lesions status more accurately is, however, a hypothesis that should be tested in further clinical studies as well as the best strategy to combine both methods (in parallel or using a sequential approach). If these points are clarified, the difference between LF readings after different air-drying times can be a promising alternative to help in caries activity assessment of noncavitated caries, since the device is not able to perform that by itself. In addition, one objective method could be used in caries activity assessment, for which only the visual inspection are available at the moment.

In conclusion, LF can distinguish cavitated active and inactive lesions classified by NY, but not by LAA-ICDAS; however, this difference might be related to the visual system rather than to LF. The air-drying time could be an alternative to improve the caries activity assessment; however, longer air-drying time is suggested to be tested subsequently.

\section{References}

1. B. Nyvad and O. Fejerskov, "Assessing the stage of caries lesion activity on the basis of clinical and microbiological examination," Community Dent. Oral Epidemiol. 25(1), 69-75 (1997).

2. N. B. Pitts, "Clinical diagnosis of dental caries: a European perspective," J. Dent. Educ. 65(10), 972-978 (2001).

3. J. D. Bader and D. A. Shugars, "A systematic review of the performance of a laser fluorescence device for detecting caries," J. Am. Dent. Assoc. 135(10), 1413-1426 (2004).

4. R. Hibst, R. Paulus, and A. Lussi, "Detection of occlusal caries by laser fluorescence: basic and clinical investigations," Med. Laser Appl. 16, 205-213 (2001).

5. F. M. Mendes, S. L. Pinheiro, and A. L. Bengtson, "Effect of alteration in organic material of the occlusal caries on DIAGNOdent readings," Braz. Oral Res. 18(2), 141-144 (2004).

6. W. Buchalla, "Comparative fluorescence spectroscopy shows differences in noncavitated enamel lesions," Caries Res. 39(2), 150-156 (2005).

7. C. Pinelli, M. Campos Serra, and L. de Castro Monteiro Loffredo, "Validity and reproducibility of a laser fluorescence system for detecting the activity of white-spot lesions on free smooth surfaces in vivo," Caries Res. 36(1), 19-24 (2002).

8. V. Anttonen, L. Seppa, and H. Hausen, "Clinical study of the use of the laser fluorescence device DIAGNOdent for detection of occlusal caries in children," Caries Res. 37(1), 17-23 (2003).

9. V. Anttonen, L. Seppa, and H. Hausen, "A follow-up study of the use of DIAGNOdent for monitoring fissure caries in children," Сотти- 
nity Dent. Oral Epidemiol. 32(4), 312-318 (2004).

10. K. R. Ekstrand, D. N. J. Ricketts, E. A. M. Kidd, V. Qvist, and S. Schou, "Detection, diagnosing, monitoring, and logical treatment of occlusal caries in relation to lesion activity and severity: an in vivo examination with histological validation," Caries Res. 32(4), 247 254 (1998).

11. B. Nyvad, V. Machiulskiene, and V. Baelum, "Reliability of a new caries diagnostic system differentiating between active and inactive caries lesions," Caries Res. 33(4), 252-260 (1999).

12. D. F. Cortes, R. P. Ellwood, and K. R. Ekstrand, "An in vitro comparison of a combined FOTI/visual examination of occlusal caries with other caries diagnostic methods and the effect of stain on their diagnostic performance," Caries Res. 37(1), 8-16 (2003).

13. P. Francescut and A. Lussi, "Correlation between fissure discoloration, Diagnodent measurements, and caries depth: an in vitro study," Pediatr. Dent. 25(6), 559-564 (2003).

14. F. M. Mendes, M. Hissadomi, and J. C. P. Imparato, "Effects of drying time and the presence of plaque on the in vitro performance of laser fluorescence in occlusal caries of primary teeth," Caries Res. 38(2), 104-108 (2004).

15. S. Al-Khateeb, R. A. Exterkate, E. de Josselin de Jong, B. AngmarMånsson, and J. M. ten Cate, "Light-induced fluorescence studies on dehydration of incipient enamel lesions," Caries Res. 36(1), 25-30 (2002).

16. M. Ando, G. K. Stookey, and D. T. Zero, "Ability of quantitative light-induced fluorescence (QLF) to assess the activity of white spot lesions during dehydration," Am. J. Dent. 19(1), 15-18 (2006).

17. K. R. Ekstrand, S. Martignon, D. J. Ricketts, and V. Qvist, "Detection and activity assessment of primary coronal caries lesions: a methodologic study," Oper. Dent. 32(3), 225-235 (2007).

18. M. M. Braga, F. M. Mendes, S. Martignon, D. N. Ricketts, and K. R. Ekstrand, "In vitro comparison of Nyvad's system and ICDAS-II with lesion activity assessment for evaluation of severity and activity of occlusal caries lesions in primary teeth," Caries Res. 43(5), 405412 (2009).

19. K. Murakami, Y. Kitasako, M. F. Burrow, and J. Tagami, "In vitro $\mathrm{pH}$ analysis of active and arrested dentinal caries in extracted human teeth using a micro pH sensor," Dent. Mater. J. 25(3), 423-429 (2006).

20. P. Francescut, B. Zimmerli, and A. Lussi, "Influence of different storage methods on laser fluorescence values: a two-year study," Caries Res. 40(3), 181-185 (2006).

21. A. B. MacGregor, "The extent and distribution of acid in carious dentine," Proc. R. Soc. Med. 55, 23-26 (1962).

22. M. C. Downer, "Concurrent validity of an epidemiological diagnostic system for caries with the histological appearance of extracted teeth as validating criterion," Caries Res. 9(3), 231-246 (1975).

23. A. Andersson, K. Sköld-Larsson, A. Hallgren, L. G. Petersson, and S. Twetman, "Measurement of enamel lesion regression with a laser fluorescence device (DIAGNOdent): a pilot study," Orthodontics 1(3), 201-206 (2004).

24. M. M. Braga, K. R. Ekstrand, S. Martignon, J. C. P. Imparato, D. N. J. Ricketts, and F. M. Mendes, "Clinical performance of two visual scoring criteria in detecting and assessing activity status of occlusal caries in primary teeth," Caries Res. 44(3), 300-308 (2010).

25. K. R. Ekstrand, "Improving clinical visual detection-potential for caries clinical trials," J. Dent. Res. 83(Spec. No. C), C67-71 (2004).

26. D. N. Ricketts, K. R. Ekstrand, E. A. Kidd, and T. Larsen, "Relating visual and radiographic ranked scoring systems for occlusal caries detection to histological and microbiological evidence," Oper. Dent. 27(3), 231-237 (2002)

27. C. L. Darling, G. D. Huynh, and D. Fried, "Light scattering properties of natural and artificially demineralized dental enamel at 1310 nm," J. Biomed. Opt. 11(3), 034023 (2006).

28. B. Nyvad, "Diagnosis versus detection of caries," Caries Res. 38(3), 192-198 (2004). 